\title{
A Note on Certain Modular Equations about Infinite Products of Ramanujan
}

\author{
Hong-Cun Zhai \\ Mathematics College, LuoYang Normal University, LuoYang 471022, China \\ Correspondence should be addressed to Hong-Cun Zhai; zhai_hc@163.com \\ Received 17 October 2013; Revised 8 November 2013; Accepted 8 November 2013 \\ Academic Editor: Ming Li
}

Copyright (C) 2013 Hong-Cun Zhai. This is an open access article distributed under the Creative Commons Attribution License, which permits unrestricted use, distribution, and reproduction in any medium, provided the original work is properly cited.

Ramanujan proposed additive formulae of theta functions that are related to modular equations about infinite products. Employing these formulaes, we derived some identities on infinite products. In the same spirit, we also could present elementary and simple proofs of certain Ramanujan's modular equations on infinite products.

\section{Introduction}

Q-theory is undoubtedly one of the most famous and useful mathematical theorems, such as Andrews-Askey type integral [1]

$$
\begin{aligned}
\int_{c}^{d} \frac{(q t / c, q t / d, f t, r s t ; q)_{\infty}}{(a t, b t, e t, s t ; q)_{\infty}} \phi_{2}\left[\begin{array}{c}
r, b t, \frac{c}{g} \\
r s t, b c ;
\end{array}, s g\right] d_{q} t \\
=\frac{d(1-q)(q, c / d, q d / c, a b c d, d f, r s d ; q)_{\infty}}{(a c, b c, a d, b d, s d, d e ; q)_{\infty}} \\
\times \sum_{k=0}^{\infty} \frac{(f / e, a d, s d, b d ; q)_{k}(c e)^{k}}{(q, d f, r s d, a b c d ; q)_{k}} \\
\times{ }_{3} \phi_{2}\left[\begin{array}{c}
r, b d q^{k}, \frac{a c d q^{k}}{g} \\
r s d q^{k}, a b c d q^{k} ;
\end{array}\right],
\end{aligned}
$$

Askey-Roy type integral [2]

$$
\begin{aligned}
\int_{-\pi}^{\pi}( & \left(P_{n}\left(e^{i \theta}, f\right) P_{m}\left(e^{i \theta}, g\right)\right. \\
& \left.\times\left(\frac{\rho e^{i \theta}}{d}, q d e^{-i \theta}, \rho c e^{-i \theta}, \frac{q e^{i \theta}}{(c \rho)} ; q\right)_{\infty}\right)
\end{aligned}
$$

$$
\begin{aligned}
& \left.\times\left(\left(a e^{i \theta}, b e^{i \theta}, c e^{-i \theta}, d e^{-i \theta} ; q\right)_{\infty}\right)^{-1}\right) d \theta \\
= & \frac{2 \pi(a f ; q)_{n}(b g ; q)_{m}(a b c d, \rho c / d, d q /(\rho c), \rho, q / \rho ; q)_{\infty}}{a^{n} b^{m}(q, b c, b d, a c, a d ; q)_{\infty}} \\
& \times \sum_{k=0}^{n} \frac{\left(q^{-n}, a c, a d ; q\right)_{k} q^{k}}{(q, a f, a b c d ; q)_{k}}{ }_{3} \phi_{2}\left[\begin{array}{c}
q^{-m}, b c, b d \\
b g, a b c d q^{k} ;
\end{array}, q\right],
\end{aligned}
$$

Moment integrals [3]

$$
\begin{aligned}
\int_{-\infty}^{\infty} & \frac{P_{n}(w, c) P_{m}(w, d)}{(a w, b w ; q)_{\infty}} d \alpha^{(s, t)}(w) \\
= & \frac{(a c ; q)_{n}(b d ; q)_{m}(a b s t ; q)_{\infty}}{a^{n} b^{m}(a s, a t, b s, b t ; q)_{\infty}} \\
& \times \sum_{k=0}^{n} \frac{\left(q^{-n}, a s, a t ; q\right)_{k} q^{k}}{(q, a c, a b s t ; q)_{k}}{ }_{3} \phi_{2}\left[\begin{array}{c}
q^{-m}, b s, b t \\
b d, a b s t q^{k} ; q, q
\end{array}\right],
\end{aligned}
$$

(where $\left.P_{n}(a, b)=(a-b) \cdots\left(a-b q^{n-1}\right)\right), q$-Fractional Calculus Equations [4] and $q$-Calculus [5]. For more information, please refer to [1-5].

The theta functions are very useful tool in researching $q$-series, especially in dealing with the form of the equation similar to above formulas, whose left-hand side is summation and right-hand side is integral. The additive identities of 
theta are one of the important of Ramanujan's contributions. Using it, we gave elementary and simple proofs of certain Ramanujan's modular equations on infinite products. For more information, please refer to [1-7].

In his notebook [8, pages 34-38], Ramanujan defines the following theta functions:

$$
\begin{aligned}
f(a, b) & :=\sum_{n=-\infty}^{\infty} a^{n(n+1) / 2} b^{n(n-1) / 2} \\
& =(-a ; a b)_{\infty}(-b ; a b)_{\infty}(a b ; a b)_{\infty}, \quad|a b|<1, \\
\varphi(q):=f(q, q) & =\sum_{n=-\infty}^{\infty} q^{n^{2}}=\frac{(-q ;-q)_{\infty}}{(q ;-q)_{\infty}}, \\
\psi(q) & :=f\left(q, q^{3}\right)=\sum_{n=1}^{\infty} q^{n(n-1) / 2}=\frac{\left(q^{2} ; q^{2}\right)_{\infty}}{\left(q ; q^{2}\right)_{\infty}},
\end{aligned}
$$

where

$$
(a ; q)_{\infty}:=\prod_{n=0}^{\infty}\left(1-a q^{n}\right), \quad|q|<1,
$$

sometimes written as

$$
(a, b, c, \ldots ; q)_{\infty}=(a ; q)_{\infty}(b ; q)_{\infty}(c ; q)_{\infty} \cdots .
$$

The infinite products are from the Jacobi triple product identity [8, page 35$]$.

In the course of deduction, we used the following simple fact $[9,10]$ :

$$
\begin{aligned}
& \left(a^{k} ; q^{k}\right)_{\infty}=\left(a, a \omega_{k}, \ldots, a \omega_{k}^{k-1} ; q\right)_{\infty} \\
& (a, q)_{\infty}=\left(a, a q, a q^{2}, \ldots, a q^{k-1} ; q^{k}\right)_{\infty} .
\end{aligned}
$$

By definition of Ramanujan theta functions one can easily verify the following identities [8, page 45]:

$$
\begin{gathered}
f(a, b)+f(-a,-b)=2 f\left(a^{3} b, a b^{3}\right), \\
f(a, b)-f(-a,-b)=2 a f\left(\frac{b}{a}, \frac{a}{b} a^{4} b^{4}\right) .
\end{gathered}
$$

From (4), if $a b=c d=p$, we have

$$
f(a, b) f(c, d)=\sum_{m, n=-\infty}^{\infty} p^{\left(m^{2}+n^{2}\right) / 2-(m+n) / 2} a^{m} c^{n} .
$$

Thus setting $m-n=2 j$ and $m+n=2 k$, we find that

$$
\begin{gathered}
f(a, b) f(c, d)+f(-a,-b) f(-c,-d) \\
=\sum_{\substack{n, m=-\infty \\
m, n, \text { even }}}^{+\infty} p^{\left(m^{2}+n^{2}\right) / 2-(m+n) / 2} a^{m} c^{n} \\
=2 \sum_{j, k=-\infty}^{\infty} p^{j^{2}+k^{2}-k} a^{j+k} c^{k-j} \\
=2 \sum_{j, k=-\infty}^{\infty} p^{k(k-1)}(a c)^{k} p^{j(j-1)}(b c)^{-j} \\
=2 f(a c, b d) f(a d, b c) .
\end{gathered}
$$

Thus when $a b=c d$, we have

$$
\begin{aligned}
& f(a, b) f(c, d)+f(-a,-b) f(-c,-d) \\
& \quad=2 f(a c, b d) f(a d, b c) .
\end{aligned}
$$

Similarly we have that

$$
\begin{gathered}
f(a, b) f(c, d)-f(-a,-b) f(-c,-d) \\
=2 a f\left(\frac{b}{c}, \frac{a b c d}{b / c}\right) f\left(\frac{b}{d}, \frac{a b c d}{b / d}\right) .
\end{gathered}
$$

The special case of these identities can be written as the following form by using Jacobian theta function $[6,7]$ :

$$
\begin{aligned}
2 \theta_{1} & (x+y \mid 2 \tau) \theta_{1}(x-y \mid 2 \tau) \\
& =\theta_{3}(y \mid \tau) \theta_{4}(x \mid \tau)-\theta_{3}(x \mid \tau) \theta_{4}(y \mid \tau) .
\end{aligned}
$$

The authors of $[6,7]$ give simple proofs and very important use of it.

In the above two identities, putting $c=a$ and $d=b$, we easily obtain

$$
\begin{gathered}
f^{2}(a, b)+f^{2}(-a,-b)=2 f\left(a^{2}, b^{2}\right) \varphi(a b), \\
f^{2}(a, b)-f^{2}(-a,-b)=4 a f\left(\frac{b}{a}, \frac{a}{b} a^{2} b^{2}\right) \psi\left(a^{2} b^{2}\right) .
\end{gathered}
$$

\section{Main Results}

The sums and products of infinite are used in many domains of mathematics, such as Partition Functions [11-14], Fractal Geometry [9], Fractional Calculus [10], Fractal Time Series [4], and so on. Then the equations of it are concentrated by several mathematicians and engineers [15-18]. At the same time, it can be used in dynamic equations, differential equations [19], and partial differential equations [20].

This paper has two main purposes. The first is to derive the identities as follows: for $|q|<1$,

$$
\begin{aligned}
& \frac{(q ; q)_{\infty}^{2}}{\left(q^{3} ; q^{3}\right)_{\infty}^{2}}+3 \frac{(-q ; q)_{\infty}^{2}}{\left(-q^{3} ; q^{3}\right)_{\infty}^{2}} \\
& \quad=4 \omega \frac{(q ; q)_{\infty}^{2}}{\left(q^{3} ; q^{3}\right)_{\infty}^{2}} \frac{f\left(q \omega, q^{3} \omega^{2}\right) f\left(q \omega^{2}, q^{3} \omega\right)}{f^{2}\left(\omega, q \omega^{2}\right)} \\
& \frac{(q ; q)_{\infty}^{2}}{\left(q^{3} ; q^{3}\right)_{\infty}^{2}}-3 \frac{(-q ; q)_{\infty}^{2}}{\left(-q^{3} ; q^{3}\right)_{\infty}^{2}}=2 \frac{(q ; q)_{\infty}^{2}}{\left(q^{3} ; q^{3}\right)_{\infty}^{2}} \frac{f\left(\omega^{2}, q \omega\right)}{f^{2}\left(\omega, q \omega^{2}\right)} \varphi(q)
\end{aligned}
$$




$$
\begin{aligned}
& \frac{(q ; q)_{\infty}^{2}}{\left(q^{5} ; q^{5}\right)_{\infty}^{2}}-5 \frac{(-q ; q)_{\infty}^{2}}{\left(-q^{5} ; q^{5}\right)_{\infty}^{2}} \\
& =4 \zeta \frac{(q ; q)_{\infty}^{2}}{\left(q^{5} ; q^{5}\right)_{\infty}^{2}} \\
& \quad \times \frac{f\left(q \zeta^{2}, q \zeta^{3}\right) f\left(\zeta, q^{2} \zeta^{4}\right) f\left(\zeta^{3}, q^{2} \zeta^{2}\right) f\left(q \zeta, q \zeta^{4}\right)}{f^{2}\left(\zeta, q \zeta^{4}\right) f^{2}\left(\zeta^{2}, q \zeta^{3}\right)},
\end{aligned}
$$

in which $\omega=\exp (2 i \pi / 3)$ and $\zeta=\exp (2 i \pi / 5)$. In the same way, we are able to give the simple and elementary proofs of the following identities of Ramanujan $[8,11,12]$ :

$$
\frac{(-q ; q)_{\infty}^{2}}{\left(-q^{3} ; q^{3}\right)_{\infty}^{2}}+\frac{(q ; q)_{\infty}^{2}}{\left(q^{3} ; q^{3}\right)_{\infty}^{2}}=2 \frac{\left(-q^{3} ; q^{6}\right)_{\infty}^{2}}{\left(q^{3} ; q^{6}\right)_{\infty}^{2}}\left(-q^{2},-q^{4} ; q^{6}\right)_{\infty}
$$$$
\frac{(-q ; q)_{\infty}^{2}}{\left(-q^{3} ; q^{3}\right)_{\infty}^{2}}-\frac{(q ; q)_{\infty}^{2}}{\left(q^{3} ; q^{3}\right)_{\infty}^{2}}=4 q \frac{\left(-q^{6} ; q^{6}\right)_{\infty}^{2}}{\left(q^{3} ; q^{6}\right)_{\infty}^{2}}\left(-q,-q^{5} ; q^{6}\right)_{\infty}
$$$$
\frac{(-q ; q)_{\infty}^{2}}{\left(-q^{5} ; q^{5}\right)_{\infty}^{2}}-\frac{(q ; q)_{\infty}^{2}}{\left(q^{5} ; q^{5}\right)_{\infty}^{2}}
$$$$
=4 q \frac{\left(-q,-q^{2},-q^{3},-q^{4},-q^{6},-q^{7},-q^{8},-q^{9} ; q^{10}\right)_{\infty}}{\left(q^{5} ; q^{10}\right)_{\infty}^{4}} .
$$

\section{Modular Equations of Infinite Productions}

In this section, we first give the two sets refinement about the identities (18) and (20).

Theorem 1. For $|q|<1$,

$$
\begin{aligned}
& \frac{(q ; q)_{\infty}}{\left(q^{3} ; q^{3}\right)_{\infty}}-i \sqrt{3} \frac{(-q ; q)_{\infty}}{\left(-q^{3} ; q^{3}\right)_{\infty}}=2 \omega \frac{(q ; q)_{\infty}}{\left(q^{3} ; q^{3}\right)_{\infty}} \frac{f\left(q \omega, q^{3} \omega^{2}\right)}{f\left(\omega, q \omega^{2}\right)} \\
& \frac{(q ; q)_{\infty}}{\left(q^{3} ; q^{3}\right)_{\infty}}+i \sqrt{3} \frac{(-q ; q)_{\infty}}{\left(-q^{3} ; q^{3}\right)_{\infty}}=2 \frac{(q ; q)_{\infty}}{\left(q^{3} ; q^{3}\right)_{\infty}} \frac{f\left(q \omega^{2}, q^{3} \omega\right)}{f\left(\omega, q \omega^{2}\right)}
\end{aligned}
$$

Proof. Note that $1+\omega+\omega^{2}=0$ and $\omega-\omega^{2}=i \sqrt{3}$. By (9), we get that

$$
\begin{aligned}
i \sqrt{3} \frac{(-q ; q)_{\infty}}{\left(-q^{3} ; q^{3}\right)_{\infty}} \div \frac{(q ; q)_{\infty}}{\left(q^{3} ; q^{3}\right)_{\infty}} \\
\quad=\frac{\left(\omega-\omega^{2}\right)(q \omega ; q)_{\infty}\left(q \omega^{2} ; q\right)_{\infty}}{(-q \omega ; q)_{\infty}\left(-q \omega^{2} ; q\right)_{\infty}}
\end{aligned}
$$

$$
\begin{aligned}
& =\frac{\omega(1-\omega)(1+\omega)(q \omega ; q)_{\infty}\left(q \omega^{2} ; q\right)_{\infty}}{(1+\omega)(-q \omega ; q)_{\infty}\left(-q \omega^{2} ; q\right)_{\infty}} \\
& =-\frac{(\omega ; q)_{\infty}\left(q \omega^{2} ; q\right)_{\infty}}{(-\omega ; q)_{\infty}\left(-q \omega^{2} ; q\right)_{\infty}} \\
& =-\frac{f\left(-\omega,-q \omega^{2}\right)}{f\left(\omega, q \omega^{2}\right)} .
\end{aligned}
$$

From (10), we have

$$
\begin{aligned}
& f(\omega, q \omega)-f(-\omega,-q \omega)=2 f\left(q \omega^{2}, q^{3} \omega\right), \\
& f(\omega, q \omega)+f(-\omega,-q \omega)=2 \omega f\left(q \omega, q^{3} \omega^{2}\right) .
\end{aligned}
$$

Dividing by $f(\omega, q \omega)$, respectively, and then applying (25), we derive

$$
\begin{aligned}
& i \sqrt{3} \frac{(-q ; q)_{\infty}}{\left(-q^{3} ; q^{3}\right)_{\infty}} \div \frac{(q ; q)_{\infty}}{\left(q^{3} ; q^{3}\right)_{\infty}}-1 \\
& =-1-\frac{f\left(-\omega,-q \omega^{2}\right)}{f\left(\omega, q \omega^{2}\right)}=-2 \omega \frac{f\left(q \omega, q^{3} \omega^{2}\right)}{f\left(\omega, q \omega^{2}\right)} \\
& i \sqrt{3} \frac{(-q ; q)_{\infty}}{\left(-q^{3} ; q^{3}\right)_{\infty}} \div \frac{(q ; q)_{\infty}}{\left(q^{3} ; q^{3}\right)_{\infty}}+1 \\
& =1-\frac{f\left(-\omega,-q \omega^{2}\right)}{f\left(\omega, q \omega^{2}\right)}=2 \frac{f\left(q \omega^{2}, q^{3} \omega\right)}{f\left(\omega, q \omega^{2}\right)} .
\end{aligned}
$$

Multiplying by $(q ; q)_{\infty} /\left(q^{3} ; q^{3}\right)_{\infty}$, respectively, we complete the proofs of (24).

Proof of (19). Let $\alpha=\exp (i \pi / 6)$; then it is easy to know that $\omega=i \alpha$ and $\alpha+1 / \alpha=-\sqrt{3}$.

One has

$$
\begin{aligned}
& \sqrt{3} \frac{(-q ; q)_{\infty}}{\left(-q^{3} ; q^{3}\right)_{\infty}} \div \frac{(q ; q)_{\infty}}{\left(q^{3} ; q^{3}\right)_{\infty}} \\
& \quad=-\left(\alpha+\frac{1}{\alpha}\right) \frac{(q \omega ; q)_{\infty}\left(q \omega^{2} ; q\right)_{\infty}}{(-q \omega ; q)_{\infty}\left(-q \omega^{2} ; q\right)_{\infty}} \\
& \quad=-\frac{1}{\alpha}\left(1+\alpha^{2}\right) \frac{(q \omega ; q)_{\infty}\left(q \omega^{2} ; q\right)_{\infty}}{(-q \omega ; q)_{\infty}\left(-q \omega^{2} ; q\right)_{\infty}} \\
& =-\frac{1}{\alpha}(1-i \alpha)(1+i \alpha) \frac{(q \omega ; q)_{\infty}\left(q \omega^{2} ; q\right)_{\infty}}{(-q \omega ; q)_{\infty}\left(-q \omega^{2} ; q\right)_{\infty}} \\
& =-\frac{1}{\alpha}(1-\omega)(1+\omega) \frac{(q \omega ; q)_{\infty}\left(q \omega^{2} ; q\right)_{\infty}}{(-q \omega ; q)_{\infty}\left(-q \omega^{2} ; q\right)_{\infty}}
\end{aligned}
$$




$$
\begin{aligned}
& =-\frac{1}{\alpha}(1+\omega)^{2} \frac{(\omega ; q)_{\infty}\left(q \omega^{2} ; q\right)_{\infty}}{(-\omega ; q)_{\infty}\left(-q \omega^{2} ; q\right)_{\infty}} \\
& =-\frac{\omega}{\alpha} \frac{f\left(-\omega,-q \omega^{2}\right)}{f\left(\omega, q \omega^{2}\right)}=-i \frac{f\left(-\omega,-q \omega^{2}\right)}{f\left(\omega, q \omega^{2}\right)} .
\end{aligned}
$$

Then we obtain that

$$
\begin{gathered}
1+\sqrt{3} \frac{(-q ; q)_{\infty}}{\left(-q^{3} ; q^{3}\right)_{\infty}} \div \frac{(q ; q)_{\infty}}{\left(q^{3} ; q^{3}\right)_{\infty}}=1-i \frac{f\left(-\omega,-q \omega^{2}\right)}{f\left(\omega, q \omega^{2}\right)}, \\
1-\sqrt{3} \frac{(-q ; q)_{\infty}}{\left(-q^{3} ; q^{3}\right)_{\infty}} \div \frac{(q ; q)_{\infty}}{\left(q^{3} ; q^{3}\right)_{\infty}}=1+i \frac{\left(-\omega,-q \omega^{2}\right)}{f\left(\omega, q \omega^{2}\right)} .
\end{gathered}
$$

In (16), let $a=\omega$ and $b=q \omega^{2}$ then we have that

$$
f^{2}\left(\omega, q \omega^{2}\right)+f^{2}\left(-\omega,-q \omega^{2}\right)=2 f\left(\omega^{2}, q^{2} \omega\right) \varphi(q) .
$$

Dividing by $f^{2}(\omega, q \omega)$, respectively, we arrive at

$$
1+\frac{f^{2}\left(-\omega,-q \omega^{2}\right)}{f^{2}\left(\omega, q \omega^{2}\right)}=\frac{2 f\left(\omega^{2}, q^{2} \omega\right) \varphi(q)}{f^{2}\left(\omega, q \omega^{2}\right)} .
$$

Multiplying (31), combining with (33), and then multiplyed by $(q ; q)_{\infty}^{2} /\left(q^{3} ; q^{3}\right)_{\infty}^{2}$, we are able to obtain (19).

Theorem 2. For $|q|<1$,

$$
\begin{aligned}
& \frac{(q ; q)_{\infty}}{\left(q^{5} ; q^{5}\right)_{\infty}}-\sqrt{5} \frac{(-q ; q)_{\infty}}{\left(-q^{5} ; q^{5}\right)_{\infty}} \\
& =2 \zeta \frac{(q ; q)_{\infty}}{\left(q^{5} ; q^{5}\right)_{\infty}} \frac{f\left(q \zeta^{2}, q \zeta^{3}\right) f\left(\zeta, q^{2} \zeta^{4}\right)}{f\left(\zeta, q \zeta^{4}\right) f\left(\zeta^{2}, q \zeta^{3}\right)} \\
& \frac{(q ; q)_{\infty}}{\left(q^{5} ; q^{5}\right)_{\infty}}+\sqrt{5} \frac{(-q ; q)_{\infty}}{\left(-q^{5} ; q^{5}\right)_{\infty}} \\
& =2 \frac{(q ; q)_{\infty}}{\left(q^{5} ; q^{5}\right)_{\infty}} \frac{f\left(\zeta^{3}, q^{2} \zeta^{2}\right) f\left(q \zeta, q \zeta^{4}\right)}{f\left(\zeta, q \zeta^{4}\right) f\left(\zeta^{2}, q \zeta^{3}\right)}
\end{aligned}
$$

Proof. First we recall that $\zeta^{5}=1, \zeta+\zeta^{4}-\zeta^{2}-\zeta^{3}=\sqrt{5}$ and $1+\zeta+\zeta^{2}+\zeta^{3}+\zeta^{4}=0$. Using (9), we have

$$
\begin{aligned}
\sqrt{5} \frac{(-q ; q)_{\infty}}{\left(-q^{5} ; q^{5}\right)_{\infty}} \div \frac{(q ; q)_{\infty}}{\left(q^{5} ; q^{5}\right)_{\infty}} \\
=\left(\zeta+\zeta^{4}-\zeta^{2}-\zeta^{3}\right) \\
\quad \times \frac{(q \zeta ; q)_{\infty}\left(q \zeta^{2} ; q\right)_{\infty}\left(q \zeta^{3} ; q\right)_{\infty}\left(q \zeta^{4} ; q\right)_{\infty}}{(-q \zeta ; q)_{\infty}\left(-q \zeta^{2} ; q\right)_{\infty}\left(-q \zeta^{3} ; q\right)_{\infty}\left(-q \zeta^{4} ; q\right)_{\infty}}
\end{aligned}
$$

$$
\begin{aligned}
= & \left(\zeta(1-\zeta)\left(1-\zeta^{2}\right)(1+\zeta)\left(1+\zeta^{2}\right)\right. \\
& \left.\times(q \zeta ; q)_{\infty}\left(q \zeta^{2} ; q\right)_{\infty}\left(q \zeta^{3} ; q\right)_{\infty}\left(q \zeta^{4} ; q\right)_{\infty}\right) \\
\times & \left((1+\zeta)\left(1+\zeta^{2}\right)(-q \zeta ; q)_{\infty}\right. \\
& \left.\quad \times\left(-q \zeta^{2} ; q\right)_{\infty}\left(-q \zeta^{3} ; q\right)_{\infty}\left(-q \zeta^{4} ; q\right)_{\infty}\right)^{-1} \\
= & -\frac{f\left(-\zeta,-q \zeta^{4}\right) f\left(-\zeta^{2},-q \zeta^{3}\right)}{f\left(\zeta, q \zeta^{4}\right) f\left(\zeta^{2}, q \zeta^{3}\right)} .
\end{aligned}
$$

Then we know easily that

$$
\begin{aligned}
1+ & \sqrt{5} \frac{(-q ; q)_{\infty}}{\left(-q^{5} ; q^{5}\right)_{\infty}} \div \frac{(q ; q)_{\infty}}{\left(q^{5} ; q^{5}\right)_{\infty}} \\
= & 1-\frac{f\left(-\zeta,-q \zeta^{4}\right) f\left(-\zeta^{2},-q \zeta^{3}\right)}{f\left(\zeta, q \zeta^{4}\right) f\left(\zeta^{2}, q \zeta^{3}\right)}, \\
1- & \sqrt{5} \frac{(-q ; q)_{\infty}}{\left(-q^{5} ; q^{5}\right)_{\infty}} \div \frac{(q ; q)_{\infty}}{\left(q^{5} ; q^{5}\right)_{\infty}} \\
= & 1+\frac{f\left(-\zeta,-q \zeta^{4}\right) f\left(-\zeta^{2},-q \zeta^{3}\right)}{f\left(\zeta, q \zeta^{4}\right) f\left(\zeta^{2}, q \zeta^{3}\right)} .
\end{aligned}
$$

In (13) and (14), setting $a=\zeta, b=q \zeta^{4}, c=\zeta^{2}$, and $d=q \zeta^{3}$, we get that

$$
\begin{aligned}
& f\left(\zeta, q \zeta^{4}\right) f\left(\zeta^{2}, q \zeta^{3}\right)+f\left(-\zeta,-q \zeta^{4}\right) f\left(-\zeta^{2},-q \zeta^{3}\right) \\
& \quad=2 f\left(\zeta^{3}, q^{2} \zeta^{2}\right) f\left(q \zeta^{4}, q \zeta\right), \\
& f\left(\zeta, q \zeta^{4}\right) f\left(\zeta^{2}, q \zeta^{3}\right)-f\left(-\zeta,-q \zeta^{4}\right) f\left(-\zeta^{2},-q \zeta^{3}\right) \\
& \quad=2 \zeta f\left(q \zeta^{2}, q \zeta^{3}\right) f\left(\zeta, q^{2} \zeta^{4}\right) .
\end{aligned}
$$

Dividing the above two equations by $f\left(\zeta, q \zeta^{4}\right) f\left(\zeta^{2}, q \zeta^{3}\right)$, respectively, and then combining with (37), we obtain that

$$
\begin{aligned}
& 1+\sqrt{5} \frac{(-q ; q)_{\infty}}{\left(-q^{5} ; q^{5}\right)_{\infty}} \div \frac{(q ; q)_{\infty}}{\left(q^{5} ; q^{5}\right)_{\infty}}=2 \frac{f\left(\zeta^{3}, q^{2} \zeta^{2}\right) f\left(q \zeta, q \zeta^{4}\right)}{f\left(\zeta, q \zeta^{4}\right) f\left(\zeta^{2}, q \zeta^{3}\right)}, \\
& 1-\sqrt{5} \frac{(-q ; q)_{\infty}}{\left(-q^{5} ; q^{5}\right)_{\infty}} \div \frac{(q ; q)_{\infty}}{\left(q^{5} ; q^{5}\right)_{\infty}}=2 \zeta \frac{f\left(q \zeta^{2}, q \zeta^{3}\right) f\left(\zeta, q^{2} \zeta^{4}\right)}{f\left(\zeta, q \zeta^{4}\right) f\left(\zeta^{2}, q \zeta^{3}\right)} .
\end{aligned}
$$

Multiplied by $(q ; q)_{\infty} /\left(q^{5} ; q^{5}\right)_{\infty}$, the identities (39) and (40) become (34) and (35).

Multiplying the two refinements in Theorems 1 and 2, respectively, we obtain the identities (18) and (20). Using the same method, we can obtain refinement identities of (21) and (22) which are similar to Theorems 1 and 2; then we can deduce (21), (22), and (23) easily. The details of proofs are omitted. 
The following conclusion can be obtained easily.

Corollary 3. For $|q|<1$,

$$
\begin{aligned}
& \frac{(q ; q)_{\infty}^{4}}{\left(q^{3} ; q^{3}\right)_{\infty}^{4}}-9 \frac{(-q ; q)_{\infty}^{4}}{\left(-q^{3} ; q^{3}\right)_{\infty}^{4}} \\
& =8 \omega \frac{(q ; q)_{\infty}^{4}}{\left(q^{3} ; q^{3}\right)_{\infty}^{4}} \\
& \quad \times \frac{f\left(\omega^{2}, q \omega\right) f\left(q \omega, q^{3} \omega^{2}\right) f\left(q \omega^{2}, q^{3} \omega\right)}{f^{4}\left(\omega, q \omega^{2}\right)} \varphi(q) .
\end{aligned}
$$

\section{Acknowledgments}

This work was supported by the National Science Foundation of China, Project nos. 11071107, 11371184, and U1304103. The author would like to thank the referee and editor for many valuable comments and suggestions.

\section{References}

[1] J. Cao, "A note on $q$-integrals and certain generating functions," Studies in Applied Mathematics, vol. 131, pp. 105-118, 2013.

[2] J. Cao, "Notes on Askey-Roy integral and certain generating functions for q-polynomials," Journal of Mathematical Analysis and Applications, vol. 409, no. 1, pp. 435-445, 2014.

[3] J. Cao, "A note on moment integrals and some applications," Journal of Mathematical Analysis and Applications, vol. 410, no. 1, pp. 348-360, 2014.

[4] M. H. Annaby and Z. S. Mansour, q-Fractional Calculus and Equations, vol. 2056 of Lecture Notes in Mathematics, Springer, Heidelberg, Germany, 2012.

[5] T. Ernst, A Comprehensive Treatment q-Calculus, Springer, New York, NY, USA, 2001.

[6] Z.-G. Liu, "Addition formulas for Jacobi theta functions, Dedekind's eta function, and Ramanujan's congruences," Pacific Journal of Mathematics, vol. 240, no. 1, pp. 135-150, 2009.

[7] Z.-G. Liu and X.-M. Yang, "On the Schröter formula for theta functions," International Journal of Number Theory, vol. 5, no. 8, pp. 1477-1488, 2009.

[8] B. C. Berndt, Ramanujan's Notebooks. Part III, Springer, New York, NY, USA, 1991.

[9] C. Cattani and G. Pierro, "On the fractal geometry of DNA by the binary image analysis," Bulletin of Mathematical Biology, vol. 75, pp. 1544-1570, 2013.

[10] C. Cattani, "Fractional calculus and Shannon wavelet," Mathematical Problems in Engineering, vol. 2012, Article ID 502812, 26 pages, 2012.

[11] N. D. Baruah and B. C. Berndt, "Partition identities and Ramanujan's modular equations," Journal of Combinatorial Theory. Series A, vol. 114, no. 6, pp. 1024-1045, 2007.

[12] N. D. Baruah and B. C. Berndt, "Partition identities arising from theta function identities," Acta Mathematica Sinica, vol. 24, no. 6, pp. 955-970, 2008.

[13] R. Blecksmith, J. Brillhart, and I. Gerst, "Parity results for certain partition functions and identities similar to theta function identities," Mathematics of Computation, vol. 48, no. 177, pp. 2938, 1987.
[14] R. Blecksmith, J. Brillhart, and I. Gerst, "Some infinite product identities," Mathematics of Computation, vol. 51, no. 183, pp. 301314, 1988.

[15] M. Li, Y.-Q. Chen, J.-Y. Li, and W. Zhao, "Hölder scales of sea level," Mathematical Problems in Enginering, vol. 2012, Article ID 863707, 22 pages, 2012.

[16] M. Li, W. Zhao, and C. Cattani, "Delay bound: fractal traffic passes through network servers," Mathematical Problems in Engineering, vol. 2013, Article ID 157636, 15 pages, 2013.

[17] M. Li and W. Zhao, "On 1/ $f$ noise," Mathematical Problems in Engineering, vol. 2012, Article ID 673648, 23 pages, 2012.

[18] M. Li, "Fractal time series-a tutorial review," Mathematical Problems in Engineering, vol. 2010, Article ID 157264, 26 pages, 2010.

[19] R. Agarwal, M. Bohner, D. O’Regan, and A. Peterson, “Dynamic equations on time scales: a survey," Journal of Computational and Applied Mathematics, vol. 141, no. 1-2, pp. 1-26, 2002, Dynamic equations on time scales.

[20] M. Bohner and A. Peterson, Dynamic Equations on Time Scales, Birkhäuser, Boston, Mass, USA, 2001. 


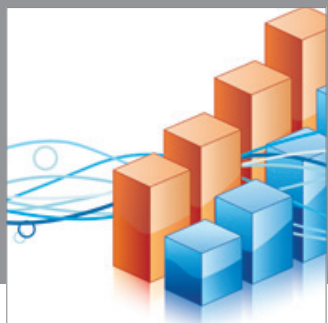

Advances in

Operations Research

mansans

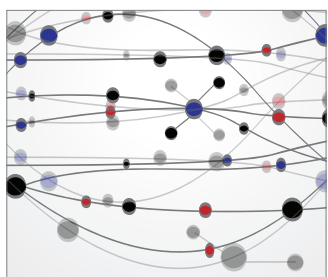

The Scientific World Journal
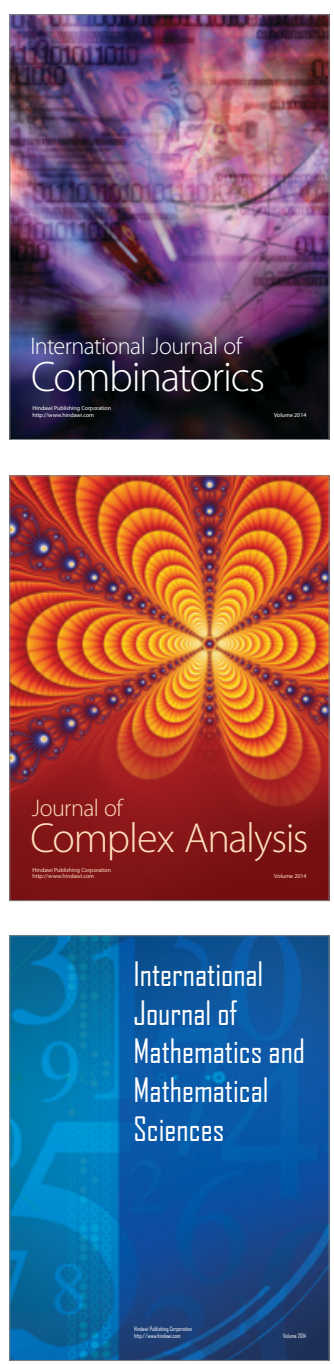
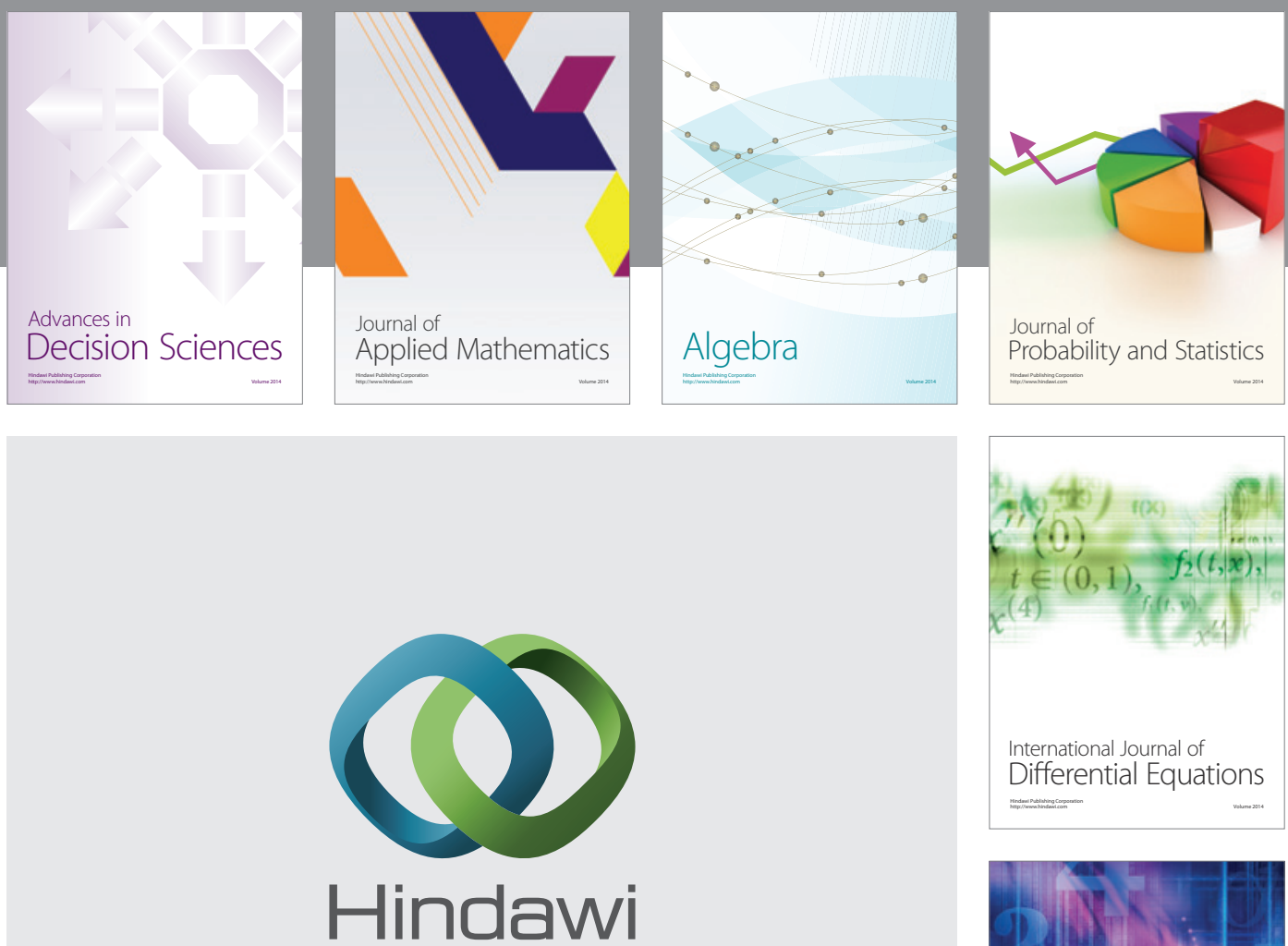

Submit your manuscripts at http://www.hindawi.com
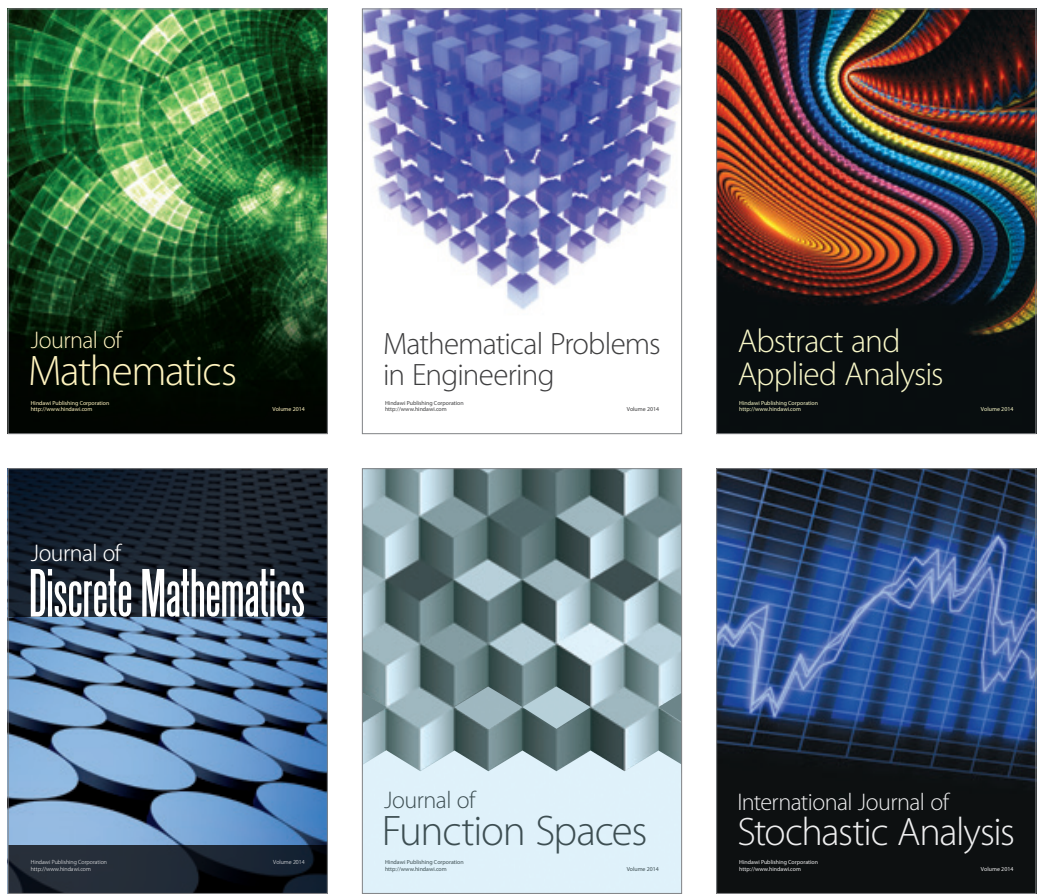

Journal of

Function Spaces

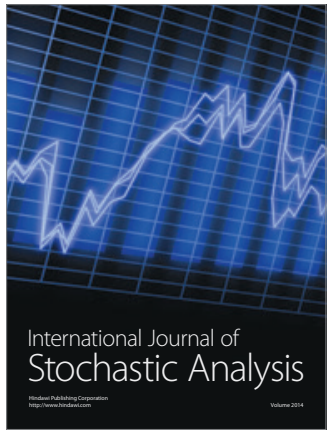

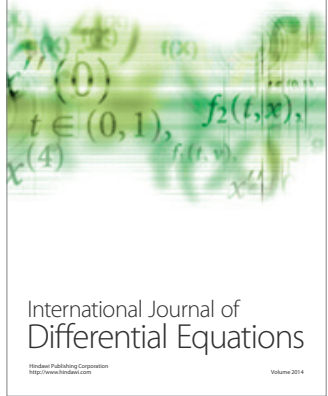
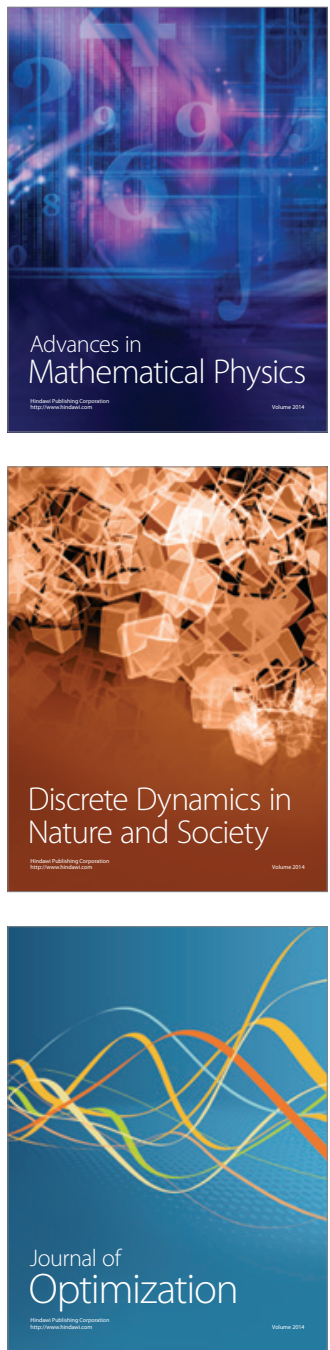